\title{
The Comparison of ISUP Grades Between Prostate Biopsy and Radical Prostatectomy: The Incoherence and Related Factors
}

\author{
Erman Ceyhan', [MD] \\ ORCID: 0000-0001-8223-6399 \\ Burak Yılmaz', [MD] \\ ORCID: 0000-0002-4242-9134 \\ Bülent Öztürk', [MD] \\ ORCID: 0000-0003-1944-1722
}

\author{
'Baskent University Faculty of Medicine, Department of \\ Urology, Konya, Turkey. \\ This study was presented in 5th Urological Surgery \\ Congress, Online (2020).

\section{Corresponding Author: Erman Ceyhan} \\ Baskent University Faculty of Medicine, Department of \\ Urology, Konya, Turkey. \\ E-mail: erman_ceyhan@yahoo.com
}

https://doi.org/10.32552/2021.ActaMedica.580

\section{n ABSTRACT Con}

Objective: To assess the incoherence rates between prostate biopsies and radical prostatectomy specimens with the use of the International Society of Urological Pathology grading system and to identify the related factors.

Materials and Methods: 89 radical prostatectomy patients were analyzed retrospectively. Patients with Gleason score $\geq 6$ were included to the study. Patients' prostate spesific antigen levels, digital rectal examination, prostate biopsy parameters, prostate cancer risk groups and final prostatectomy pathologies were examined. Gleason scores and International Society of Urological Pathology grades of prostate biopsy and prostatectomy specimens were compared. The coherence, upgrading and downgrading rates of pathologies assessed and related factors were identified.

Results: Patients' mean age was $63.1 \pm 6.0$ years. Prostate spesific antigen levels ranged from 2.8 to $114.0 \mathrm{ng} / \mathrm{mL}$ (mean:14.8 \pm 16.7 ). The mean number of cores biopsied was 10.9 \pm 3.1 . Number of patients in low, intermediate and high risk group were $27(30.3 \%), 34(38.2 \%)$ and $28(31.5 \%)$ respectively. The coherence, upgrading and downgrading rates according to International Society of Urological Pathology grading were $49.4 \%, 33.7 \%$ and $16.9 \%$ respectively. The low risk prostate cancer group showed the most coherent pathologies with the rate of $70.4 \%$ ( $p<0.05$, both for International Society of Urological Pathology grading and Gleason scoring). There was no significant relation between prostate spesific antigen level, number of cores biopsied, percentage of cancer involvement, presence of perineural invasion coherence, upgrading and downgrading. Also no significant difference found between coherent, upgrading and downgrading pathologies with respect to the time to radical prostatectomy.

Conclusion: The incoherence between prostate biopsy and radical prostatectomy is challenging. Risk of upgrading and downgrading should be considered in decision making. Low risk prostate cancer shows the most coherent pathology between prostate biopsy and radical prostatectomy.

Keywords: Biopsy, prostatectomy, neoplasm grading, prostatic neoplasms, decision making

Received: 1 April 2021, Accepted: 4 July 2021,

Published online: 28 September 2021 


\section{INTRODUCTION}

The diagnose of prostate cancer mostly starts with a digital rectal examination or prostate spesific antigen (PSA) level screening. The second step is trans rectal ultrasound (TRUS) guided biopsy which gives us the preliminary tumor grade. After the diagnosis of prostate cancer, treatment options include active surveillence, surgery, radiotherapy, hormonal therapy, chemotherapy and combinations. To choose the proper treatment method, PSA and biopsy parameters used predominantly. Considering the increasing popularity of active surveillence, a well sampled prostate biopsy is crucial in this concept [1].

The Gleason scoring has been used for a long period of time but after escaleting modifications, a simpler grading system was devised by the International Society of Urological Pathology (ISUP) [2]. The ISUP grading system comprises of 5 groups where the distiction between Gleason score 7 malignancies is defined significantly. Thus the incoherence between prostate biopsy and radical prostatectomy (RP) manifests difference in Gleason scoring and ISUP grading.

There are great number of studies in the literature reporting Gleason score disperancies between TRUS guided biopsies and RP specimens [3-5]. The fenomenon of upgrading is mostly associated to the disease heterogenity and poor sampling due to the TRUS guidence which results in higher Gleason score in RP pathology $[3,5,6]$. The downgrading is thought to be the result of oversampling the part with the worser pathology or diversity in pathological evaluation $[3,4]$.

We asessed our center's incoherence rates between prostate biopsies and RP specimens with the use of the new ISUP grading system and identified the related factors.

\section{MATERIALS and METHODS}

We retrospectively analyzed 89 patients who underwent RP in our center between 20052019. This study was approved by Institutional Review Board (Project no: KA19/409). Patients lacking prostate biopsy details and patients with Gleason score $<6$ (in biopsy or RP pathology) were excluded from the study. Patients' pre-operative digital rectal examination, serum PSA level and TRUS guided prostate biopsy parameters were evaluated. Regarding biopsy parameters; number of cores, ISUP grade group, Gleason score, presence of perineural invasion and percentage of cancer involment were recorded. Patients were stratified into three risk groups; low risk, intermediate risk and high risk according to the European Association of Urology risk groups for prostate cancer [7].

All patients underwent open retropubic RP and lymph node dissection was performed in 82 patients. Time to RP after prostate biopsy was noted. Related to RP pathology; tumor stage according to TNM (all T2 substages were merged as T2) classification, nodal involvement, ISUP grade group, Gleason score, presence of perineural invasion, capsular invasion, lymphovascular invasion and positive surgical margins were recorded. The incoherence between prostate biopsies and RP specimens were sorted separately for ISUP grading groups and Gleason scoring (3+4 and $4+3$ scores identified in different groups). Higher ISUP grade/Gleason score in RP specimen defined as "upgrading" and higher ISUP grade/Gleason score in prostate biopsy report was defined as "downgrading".

\section{Statistics}

Statistical analyses were performed by Statistics Package for Social Sciences version 25 (SPSS ${ }^{\circledR}$ v25, IBM Corp., Armonk, N.Y., USA). Continuous variables were reported by using mean ( \pm standart deviation) and median (minimum-maximum). Categorical variables were reported by using percentages. Continuous parameters between two groups with non-normal distribution were compared with Mann-Whitney $U$ test. Analyses of multiple variables were performed by One Way Anova and Kruskal Wallis in normally distributed and nonnormal distributed data respectively. Chi-square test was used for categorical data analysis. $P$ value $<0.05$ is considered statistically significant.

\section{RESULTS}

The mean age of men was $63.1 \pm 6.0$ years. Digital rectal examination was abnormal in 48/89 (53.9\%) patients. PSA levels of men ranged from 2.8 to $114.0 \mathrm{ng} / \mathrm{mL}$ (mean: $14.8 \pm 16.7$ ) The mean number 
of cores biopsied was 10.9 \pm 3.1 (min:4, max:22). Number of patients in low risk group, intermediate risk group and high risk group were 27(30.3\%), 34 (38.2\%) and 28 (31.5\%) respectively.

The mean time to RP after TRUS biopsy was $5.2 \pm 3.4$ weeks. Lymph node dissection was performed in 82 (92.1\%) patients. Pathological evaluation of RP sepecimens presented 44 (49.4\%) T2 disease, 27 (30.3\%) T3a disease and 18 (20.2\%) T3b disease. $6 / 89$ (6.7\%) patients had N1 disease. Presence of perineural invasion in RP specimen was seen in 69 (77.5\%) patients, capsular invasion was positive in $58(65.2 \%)$ patients, lymphovascular invasion was present in $15(16.9 \%)$ patients and positive surgical margins were detected in 18 (20.2\%) patients.

The pathological results of biopsies and RP specimens as stated in ISUP grade groups were listed in Table 1. The incohorence between TRUS biopsies and RP specimens according to ISUP grade groups and Gleason scores was listed in Table 2. When the Gleason score was put to use as sum of Gleason grades; the coherence, upgrading and downgrading rate changed as $59.6 \%, 29.2 \%$ and $11.2 \%$ respectively.

There was no significant association between digital rectal examination and upgrading/downgrading of ISUP grades and Gleason scores $(p=0.41$ and $p=0.23$ respectively). Also no significant relation was found between number of cores biopsied and upgrading/ downgrading of ISUP grades and the Gleason scores $(p=0.76$ and $p=0.82$ ). Regarding biopsy parameters, percentage of cancer involment and presence of perineural invasion were not different among coherent, upgrading, downgrading pathologies ( $p>0.05$ both for ISUP grade groups and Gleason scores)

PSA levels showed no significant difference among coherent, upgrading and downgrading pathologies ( $p=0.11$ for ISUP grade groups and $p=0.07$ for Gleason scores). ISUP grade group coherence of biopsy and RP specimens among risk groups was listed in Table 3. The coherence of pathology reports was significantly higher in the low risk group ( $p<0.05$ both for ISUP grade groups and Gleason scores). There was no significant difference between coherent, upgrading and downgrading pathologies with respect to the time to RP ( $p=0.24$ for ISUP grades and $p=0.14$ for Gleason scores).

Surgical margin positivity showed no significant difference in patients with upgrading pathologies $(p=0.40)$. However seminal vesicale involvement was significantly higher in upgrading cases than that of coherent and downgrading pathologies $(p<0.05)$.

PSA levels showed significant difference among $T$ stage of RP specimens with a marked difference between $T 2$ and T3b staged diseases $(p<0.05)$. Also PSA levels were significantly higher in N1 staged disease ( $p<0.05$, median 17.5 vs 8.9 ).

Patients with PSA under $10 \mathrm{ng} / \mathrm{mL}$ were evaluated seperately with respect to upgrading and downgrading pathologies. 5 out of 20 patients

Table 1. The ISUP grade groups of TRUS guided biopsies and RP specimens

\begin{tabular}{|l|c|c|c|c|c|}
\hline Biopsy ISUP Grade Group & Patients & $\%$ & RP ISUP Grade Group & Patients & $\%$ \\
\hline 1 & 48 & 53.9 & 1 & 40 & 44.9 \\
2 & 17 & 19.1 & 2 & 19 & 21.3 \\
3 & 12 & 13.5 & 3 & 11 & 12.4 \\
4 & 6 & 6.7 & 4 & 11 & 9.0 \\
\hline 5 & 6 & 6.7 & 5 & 89 & 12.4 \\
\hline Total & 89 & 100 & Total & 100 \\
\hline
\end{tabular}

Table 2. Incohorence between TRUS guided biopsies and RP specimens

\begin{tabular}{|l|c|c|c|c|}
\hline \multirow{2}{*}{} & \multicolumn{2}{|c|}{ ISUP Grading } & \multicolumn{2}{c|}{ Gleason Scoring } \\
\cline { 2 - 5 } & Patients & $\%$ & Patients & 47.2 \\
\hline Coherent & 44 & 49.4 & 42 & 34.8 \\
Upgrading & 30 & 33.7 & 31 & 18.0 \\
Downgrading & 15 & 16.9 & 16 & 100 \\
\hline Total & 89 & 100 & 89 & \\
\hline
\end{tabular}


Table 3. ISUP grade coherence of biopsy and RP sepicimens among risk groups $(p<0.05)$

\begin{tabular}{|l|c|c|c|c|}
\hline Risk Group & Coherent & Upgrading & Downgrading & Total \\
\hline Low & $19(70.4 \%)$ & $8(29.6 \%)$ & $0(0 \%)$ & $27(100 \%)$ \\
\hline Intermediate & $14(41.2 \%)$ & $12(35.3 \%)$ & $8(23.5 \%)$ & $34(100 \%)$ \\
\hline High & $11(39.3 \%)$ & $10(35.7 \%)$ & $7(25 \%)$ & $28(100 \%)$ \\
\hline
\end{tabular}

(25\%) in whom the TRUS biopsies were reported higher than ISUP grade group 1 (Gleason score >6), the final RP pathology were reported as ISUP grade group 1 (Gleason score $=6$ ). All of these patients had T2N0 disease eventually. The other way round $29.6 \%(8 / 27)$ patients with ISUP grade group 1 (Gleason score=6) disease in TRUS biopsy were upgraded in RP pathology and identified in a higher group ( 6 patients in ISUP grade group 2 and 2 patients in ISUP grade group 3 ).

\section{DISCUSSION}

The incoherence between prostate biopsy and RP pathologies have been substantiated by literature with many reports. However the underlying causes are still unclear because of the multifactorial nature of incoherences. Most of the former studies used the Gleason scoring [3-5]. The Gleason scoring system has its own flaws as sum of some Gleason grades are catogorized with the same score. Although Gleason score $3+4=7$ and $4+3=7$ are reported as same, the diseases have different prognosis. Thus the definition of unchanged Gleason score is deficient where prostate biopsy is reported as $3+4$ and RP specimen is reported as $4+3$. With the new ISUP grading focusing on prognosis, the difference between Gleason $3+4$ and $4+3$ cancers were introduced. In this study we assessed the incohorence between prostate biopsy and RP in ISUP grading context. Similar to the reported literature data, we found a marked incoherence between prostate biopsy and RP specimens. Our data showed that the low risk prostate cancer patients exibit the most coherence between prostate biopsy pathology and the RP specimen.

There are great number of suggested causes of upgrading, the so called actual grade progression until RP is less accepted. It is commonly believed that the majority of incoherence between prostate biopsy and RP pathologies are due to sampling errors [4]. Prostate cancer is a histologically heterogenious disease where most of the RP specimens contain more than one different
Gleason grades [8]. With the conventional TRUS biopsies, high grade tumors can be undersampled due to insufficient visualization. This will result in upgrading of the biopsy pathology [4]. Besides poor sampling, the variations in reporting of pathological examination also leads to upgrading [4]. Subjectivity of tumor grading is paramount when high grade tumors sampled marginally by TRUS guidance and high graded tumor zones were disrupted by pathological preparation.

The phenomenon of downgrading is more indefinite and less reported. Downgrading is mostly related to the inaccuraccy of Gleason scoring and fail in specimen processing $[5,9]$. Another factor in this topic may be the tertiary pattern. The reporting differences in tertiary pattern may contribute to downgrading.

In this study we found $47.2 \%$ coherence in pathologies by Gleason scoring. The upgrading and downgrading rates were $34.8 \%$ and $18 \%$ respectively. Bullock et al. reported higher concordance rate with $58.9 \%$ in their British registry. Whereas their upgrade and downgrade rates were lower (25.5\% and $15.6 \%$ respectively). Ooi and Samali's concordance rate was comparable to ours with $43 \%$ but their upgrading rate was higher (46\%) [10]. In the study of Dolatkhah et al. their overall undergrading (upgrading) and overgrading(downgrading) rates were $34 \%$ and $7 \%$ respectively [11]. A more standardized study of $L$. Egevad et al. they reported $45.5 \%$ coherent results. $38.8 \%$ of their patients were undergraded and $15.7 \%$ were overgraded [3]. In a study of Coogan et al. with three different number of cores $(6,8$ and 10 cores), they reported their overall coherent, upgrading and downgrading rates as $48.3 \%, 35.4 \%$ and $16.3 \%$ respectively [12]. The Lahey data presented higher coherence with $58 \%$, higher upgrading rate with $36 \%$ but lower downgrading with $5 \%$ with respect to Gleason scores (sum of grades) [5]. The metaanalysis they refered demonstrated even higher coherence rate as $63 \%$.

It is clear that by sampling more cores, the chance to detect prostate cancer increases [13]. But 
more cores does not influence upgrading and down grading always. In this study we found no relation between number of cores biopsied and pathological coherence. Some authors explain this fail of improvement in scoring accuracy with intraobserver variability as this may be the situation in this study [3]. On the contrary some authors stated that increasing the number of biopsies improves coherence in Gleason scoring [10, 14]. Coogan et al. suggested that increasing the number of cores biopsied will not effect under-grading but may improve over-grading, especially in the 10-core biopsy group [12].

Apart from the number of cores biopsied, maximum percentage of cancer in core was found related. A study showed that higher maximum percantage of cancer was an independent predictive factor for upgrading while lower percentage was predictive for downgrading [9]. However we found no relation between percentage of cancer involment and upgrading/downgrading in one-way analysis of variance.

Bullock et al. assessed upgrading and downgrading rates with respect to D'Amico risk groups similar to our study. Although they found the highest upgrade rate in low risk group (55.7\%) the upgrading was lowest in low risk group (29.6\%) in our series [4]. The highest coherence in our series was in the low risk group with $70.4 \%$ where their conherence rate in ISUP grade group 1 patients was $39.6 \%$. The findings of the British registry is assumed to be the result of high risk patient cohort they operated on. Dolatkhah et al. found most of the discrepancies in low and intermediate grade Gleason scores with a dominance of undergrading [11]. L. Egevad et al. identified Gleason score 6 biopsies the least reliable Gleason group. But we found the most concordance in the low risk group and Gleason score 6 patients in biopsies as coherence rate was the highest in this group [3]. A meta-analysis categorized and assessed concordance according to Gleason score as score $<7$, score 7 and score $>7$ groups. They found the highest concordance rate in Gleason score 7 group which might have been the result of the combining Gleason 3+4 and $4+3$ cancers in one group [5]. Two other studies found that overgrading rates were higher in Gleason score 8-10 cases [11,15]. Similarly in this study overgrading was found higher in high risk group. On the other hand their undergrading rates were higher in Gleason score $\leq 6$ cases.
The time to surgery from prostate biopsy is another issue studied in the literature. Correspondingly top our study, Freedland et al. suggested that time delay to RP was not associated with upgrading [16].

Some authors reported that higher pre-operative PSA level is associated to upgrading $[4,9]$. However in our series, PSA levels showed no association to upgrading or downgrading both for Gleason scoring and ISUP grading.

The coherence of prostate biopsy tumor grade and RP tumor grade is important because of the fact that it has great impact on decision making. Undergrading poses the risk of undertreatment while overgrading carry the risk for overtreatment. Active surveillence is in one end of this spectrum. In our data $25 \%$ of patients overgraded in needle biopsy with a PSA level under $10 \mathrm{ng} / \mathrm{mL}$ diagnosed with a ISUP grade group 1 cancer. These patients had T2NO disease so that they may suffered unneccesary lymph node dissection and lost the chance of active surveillence. Moussa et al. reported the incidence of downgrading in Gleason score $7(3+4)$ cancer to Gleason score $\leq 6$ cancer as $7.3 \%$ [9]. From the other angle, $29.6 \%$ of ISUP grade group 1 cases in our series upgraded in RP pathology. For this reason urologists should be cautious about advising active surveillence considering possibility of undergrading. In the study of L. Egevad et al., 24\% of men with reported Gleason score $<7$ upgraded to Gleason score $\geq 7$ cancer [3]. The rate of upgrading in Gleason score $<7$ cases in our series is $33.3 \%$.

In the study of Freedland et al., they found that possibility of positive surgical margins and seminal vesicle invasion are higher in upgrading cases [16]. Similarly, we found higher seminal vesical involment in upgrading cases than that of coherent and downgrading pathologies. However we found no relation between upgrading and surgical margin positivity.

The main limitation of this study besides the retrospective design is the small number of patients. Larger series may reflect the difference between ISUP grading and Gleason scoring better. Also varriations in pathological reporting is an important issue which may result in both upgrading and downgrading.

The incoherenece between TRUS guided prostate biopsy and RP pathology is a troubling issue. Until 
the standard use of better image guided biopsy modalities, urologists must be cautious about upgrading/downgrading between biopsy and the final specimen. As stated in this paper, low risk prostate cancer patients exibit the most coherence between prostate biopsy pathology and the final RP pathology. Intermediate risk and high risk patients should be handled more prudently. A good pathological work-up is important that the grading has the greatest impact on dissease course. ISUP grading should be prefered rather than Gleason score until a better classification comes forward.

\section{CONFLICT Of INTEREST}

The authors declare no conflict of interest.
[1] Thomsen FB, Brasso K, Klotz LH, et al. Active surveillance for clinically localized prostate cancer--A systematic review. J Surg Oncol. 2014; 109 (8): 830-5.

[2] Epstein JI, Egevad L, Amin MB, et al. The 2014 International Society of Urological Pathology (ISUP) consensus conference on Gleason grading of prostatic carcinoma. The American journal of surgical pathology. 2016; 40(2): 244-52.

[3] Egevad L, Norlen B, Norberg M. The value of multiple core biopsies for predicting the Gleason score of prostate cancer. BJU international. 2001; 88(7): 716-21.

[4] Bullock N, Simpkin A, Fowler S, et al. Pathological upgrading in prostate cancer treated with surgery in the United Kingdom: trends and risk factors from the British Association of Urological Surgeons Radical Prostatectomy Registry. BMC urology. 2019; 19: 94.

[5] Cohen MS, Hanley RS, Kurteva T, et al. Comparing the Gleason prostate biopsy and Gleason prostatectomy grading system: the Lahey Clinic Medical Center experience and an international meta-analysis. Eur Urol. 2008; 54(2): 371-81.

[6] Ruijter E, van Leenders G, Miller G, et al. Errors in histological grading by prostatic needle biopsy specimens: frequency and predisposing factors. The Journal of Pathology: A Journal of the Pathological Society of Great Britain and Ireland. 2000; 192(2): 229-33.

[7] EAU Guidelines E. EAU Guidelines. Edn. presented at the EAU Annual Congress Amsterdam 2020. ISBN 978-9492671-07-3. 2020.

[8] Aihara M, Wheeler TM, Ohori M, et al. Heterogeneity of prostate cancer inradical prostatectomy specimens. Urology. 1994; 43(1): 60-6.
[9] Epstein Jl, Feng Z, Trock BJ, et al. Upgrading and downgrading of prostate cancer from biopsy to radical prostatectomy: incidence and predictive factors using the modified Gleason grading system and factoring in tertiary grades. Eur Urol. 2012; 61(5): 1019-24.

[10] Ooi K, Samali R. Discrepancies in Gleason scoring of prostate biopsies and radical prostatectomy specimens and the effects of multiple needle biopsies on scoring accuracy. A regional experience in Tamworth, Australia. ANZ J Surg. 2007; 77(5): 336-8.

[11] Dolatkhah S, Mirtalebi M, Daneshpajouhnejad P, et al. Discrepancies between biopsy Gleason score and radical prostatectomy specimen Gleason score: an Iranian experience. Urology journal. 2019; 16(1): 56-61.

[12] Coogan CL, Latchamsetty KC, Greenfield J, et al. Increasing the number of biopsy cores improves the concordance of biopsy Gleason score to prostatectomy Gleason score. BJU international. 2005; 96(3): 324-7.

[13] Norberg M, Egevad L, Holmberg L, et al. The sextant protocol for ultrasound-guided core biopsies of the prostate underestimates the presence of cancer. Urology. 1997; 50(4): 562-6.

[14] Miyake H, Kurahashi T, Takenaka A, et al. Improved accuracy for predicting the Gleason score of prostate cancer by increasing the number of transrectal biopsy cores. Urol Int. 2007; 79(4): 302-6.

[15] Rajinikanth A, Manoharan M, Soloway CT, et al. Trends in Gleason score: concordance between biopsy and prostatectomy over 15 years. Urology. 2008; 72(1): 177-82.

[16] Freedland SJ, Kane CJ, Amling CL, et al. Delay of radical prostatectomy and risk of biochemical progression in men with low risk prostate cancer. J Urol. 2006; 175(4): 1298303. 